\title{
Improvement in catalytic activity and thermostability of a GH10 xylanase and its synergistic degradation of biomass with cellulase
}

Shuai You ${ }^{1,2,3}$, Chen Xie ${ }^{2,3}$, Rui Ma ${ }^{1}$, Huo-qing Huang ${ }^{1}$, Richard Ansah Herman ${ }^{2,3}$, Xiao-yun Su', Yan Ge ${ }^{2,3}$, Hui-yi Cai ${ }^{1}$, Bin Yao ${ }^{1}$, Jun Wang ${ }^{2,3^{*}}$ and Hui-ying Luo ${ }^{1 *}$

\begin{abstract}
Background: Xylanase is one of the most extensively used biocatalysts for biomass degradation. However, its low catalytic efficiency and poor thermostability limit its applications. Therefore, improving the properties of xylanases to enable synergistic degradation of lignocellulosic biomass with cellulase is of considerable significance in the field of bioenergy.

Results: Using fragment replacement, we improved the catalytic performance and thermostability of a GH10 xylanase, XyIE. Of the ten hybrid enzymes obtained, seven showed xylanase activity. Substitution of fragments, M3, M6, M9, and their combinations enhanced the catalytic efficiency (by 2.4- to fourfold) as well as the specific activity (by 1.2- to 3.3-fold) of XylE. The hybrids, XyIE-M3, XyIE-M3/M6, XylE-M3/M9, and XyIE-M3/M6/M9, showed enhanced thermostability, as observed by the increase in the $T_{50}\left(3-4.7^{\circ} \mathrm{C}\right)$ and $T_{\mathrm{m}}\left(1.1-4.7^{\circ} \mathrm{C}\right)$, and extended $t_{1 / 2}($ by $1.8-2.3 \mathrm{~h}$ ). In addition, the synergistic effect of the mutant xylanase and cellulase on the degradation of mulberry bark showed that treatment with both XyIE-M3/M6 and cellulase exhibited the highest synergistic effect. In this case, the degree of synergy reached 1.3, and the reducing sugar production and dry matter reduction increased by $148 \%$ and $185 \%$, respectively, compared to treatment with only cellulase.
\end{abstract}

Conclusions: This study provides a successful strategy to improve the catalytic properties and thermostability of enzymes. We identified several xylanase candidates for applications in bioenergy and biorefinery. Synergistic degradation experiments elucidated a possible mechanism of cellulase inhibition by xylan and xylo-oligomers.

Keywords: GH10 xylanase, Catalytic efficiency, Thermostability, Fragment replacement, Biomass degradation

\section{Highlights}

1. An innovative fragment-replacement-based rational design method was developed.

\footnotetext{
*Correspondence: wangjun@just.edu.cn; luohuiying@caas.cn ${ }^{1}$ Key Laboratory for Feed Biotechnology of the Ministry of Agriculture, Feed Research Institute, Chinese Academy of Agricultural Sciences, Beijing 100081, China

${ }^{2}$ School of Biotechnology, Jiangsu University of Science and Technology, Zhenjiang 212018, People's Republic of China

Full list of author information is available at the end of the article
}

2. The specific activity and catalytic efficiency of the mutant xylanase reached $2010 \mathrm{mg} / \mathrm{mL}$ and $2280 \mathrm{~mL} / \mathrm{s} / \mathrm{mg}$, respectively.

3. $4.2 \mu \mathrm{mol} / \mathrm{mL}$ reducing sugar was obtained from simultaneous xylanase-cellulase synergistic saccharification.

4. Comprehensive biomass utilization was realized with a tailored pretreatment and glycoside hydrolase hydrolysis strategy. 


\section{Background}

With continuous development in the global economy, people are challenged with two major social issues, environmental pollution and energy crisis. Increasing consumption of non-renewable energy has further stimulated the demand for exploring renewable energy sources [1]. Biomass is considered to be among the most promising renewable energy sources due to its energyrich reserves and low cost of production [2]. Lignocellulosic materials, such as agricultural residues, are mainly composed of cellulose, hemicellulose, and other minor components. Its robust and complex structure makes it very difficult for enzymatic hydrolysis [3]. Therefore, the synergistic degradation of lignocellulosic biomass by cellulases and hemicellulases is an excellent strategy for efficiently converting it into resourceable sugars. Xylan is the major component of hemicellulose and represents an abundant renewable source of biomass for biorefineries [4]. Due to its heterogeneous structure, the degradation of xylan requires several types of backbone and sidechain-cleaving enzymes. Xylanases are crucial enzymes that catalyze the hydrolysis of xylan backbones, and have widespread applications in animal feed, food, bioethanol, detergent, and paper pulp industries $[5,6]$.

However, it is challenging to obtain a thermostable xylanase with good catalytic efficiency for industrial purposes and has been the subject of research in several disciplines [7]. Common approaches involve mining new genetic resources from extreme environments, engineering the enzymes, and optimizing their application $[8,9]$. Several xylanases from Aspergillus fumigatus R1 [10], Streptomyces coelicolor A3 [11], Aspergillus niger [12], and Bispora sp. MEY-1 [13] have high catalytic activities of 18,000 to $38,000 \mathrm{U} / \mathrm{mg}$. There have also been some successful cases of protein engineering. For example, Wang et al. [14] enhanced the catalytic efficiency of the xylanase from Geobacillus stearothermophilus by 3.46 -fold using saturation mutagenesis and directed evolution. In addition, Wang et al. [15] enhanced the catalytic efficiency of xylanases and lichenases using oligomerization. However, there is often a trade-off between enzyme activity and stability at the level of individual mutations. In other words, enzyme rigidity is required for greater thermostability, while a flexible structure favors high catalytic activity. Therefore, mutants with increased stability often have less catalytic activity $[16,17]$. Increased enzyme activity at the cost of thermostability is not biotechnologically and practically desirable. Therefore, improving the catalytic efficiency, as well as the thermostability, of an enzyme is a research focus for high-temperature industrial applications $[18,19]$.

Domains or peptide segments substitution has been used to improve the catalytic performance of enzymes. In contrast to site-directed mutagenesis, DNA shuffling, and random mutagenesis, which require complex calculations and laborious screening, specific fragment substitutions based on amino acid sequences and structure alignment can integrate the advantages of different enzymes. For example, Zheng et al. [20] improved the catalytic activity of cellulase $\mathrm{BaCel} 5$ from Bispora by substituting the N-terminal semi-barrel with its counterpart from TeEgl5A, while Song et al. [21] improved the substrate degradation rate of GH11 xylanase NTfus by replacing the N-terminal peptide with that of a highly active Np-Xyn. However, to date, there has been no study on the improvement in the enzymatic properties of a GH10 xylanase and its catalytic mechanism using peptide segments substitution.

In this study, fragment replacement was utilized to enhance the enzymatic properties of XylE based on sequence and structure alignments. We identified the key peptide segments influencing the thermostability and catalytic efficiency of GH10 xylanase and further elucidated their mechanism of action. In addition, we investigated the synergistic effect of cellulase and its accessory enzyme, xylanase (mutants with enhanced properties), on pretreated natural agricultural waste (mulberry bark, which contains approximately $31-33 \%$ cellulose, 17-19\% hemicellulose, and 5-7\% lignin). The residual dross after enzymatic hydrolysis treatment was collected to assess the effectiveness of lignocellulosic biomass hydrolysis, and then, the surface features were observed using a scanning electron microscope (SEM) to characterize the microstructure. This study thoroughly explored the mechanism by which xylanases and cellulases work together for the degradation and saccharification of lignocellulosic biomass. We have also shown that hemicellulose, especially xylan, plays a significant role in reducing the rate of enzymatic hydrolysis, which explains to some extent why the removal of hemicellulose during hydrolysis increases the saccharification efficiency of cellulase.

\section{Results \\ Fragment identification}

The catalytic domains of XylE and thermophilic XYL10C share 53\% sequence identity (Additional file 1: Figure S1), and their crystal structures showed a common $(\alpha / \beta)_{8}$-barrel fold of GH10 xylanases [22, 23]. Two standards were used to determine the demarcation point of the sequences: (1) Each sequence retains the local secondary structure and (2) except for the $\mathrm{N}$ - and $\mathrm{C}$-terminal sequences, each sequence has a length of less than 20 amino acid residues. According to their structure alignment (Fig. 1a) and the two demarcation standards described in "Materials and methods," we identified ten fragments. The cleavage sites on XylE for fragment substitution compared with those of XYL10C are shown in Fig. 1b. 
Production, expression, and purification of all enzymes Wild-type XylE and its hybrid enzymes were produced in $P$. pastoris and purified to electrophoretic uniformity as described in "Materials and methods." With beechwood xylan as the substrate, XylE and its hybrid enzyme activities were assayed at $70{ }^{\circ} \mathrm{C}$, pH 5 for $10 \mathrm{~min}$. Except for mutants XylE-M1, XylE-M2, and XylE-M10, which displayed no activity, the other mutants displayed xylanase activity. XylE-M3, XylE-M6, and XylE-M9 showed greatly increased specific activities (1130-1310 U/mg vs. $610 \mathrm{U} /$ $\mathrm{mg}$ ) compared with that of wild-type XylE. These three peptide segments were then randomly combined to generate mutants XylE-M3/M6, XylE-M3/M9, XylE-M6/M9, and XylE-M3/M6/M9. The enzymes were produced and purified as described above. SDS-PAGE analysis showed that the purified mutants XylE-M3-M9 and the combined substitution of the key fragments had molecular masses of $43-55 \mathrm{kDa}$ as compared to their theoretical values $(\sim 37 \mathrm{kDa})$. A single band was observed for all enzymes after treatment with Endo $\mathrm{H}$, which corresponded to the theoretical molecular weight (Additional file 1: Figure S2).

\section{Effect of $\mathrm{pH}$ on the properties of XylE and its mutants}

The effects of $\mathrm{pH}$ on the activity and stability of XylE and its mutants were measured using beechwood xylan as the substrate. As shown in Fig. 2a, XylE and its mutants showed maximum activity at $\mathrm{pH} 4.5$ or 5 , and the mutants, XylE-M3, XylE-M3/M6, and XylE-M3/M9, retained higher activities than XylE at $\mathrm{pH} 3-4$ (5-66\% vs. $4-27 \%)$. The results suggested that the M3 might play a role in increasing the $\mathrm{pH}$ range. After incubation at $37{ }^{\circ} \mathrm{C}$ for $1 \mathrm{~h}$, all enzymes maintained $>70 \%$ of the highest activity at $\mathrm{pH}$ ranging from 2 to 8 , and the mutant enzymes retained more activity than the wild type (81135\% vs. 75-109\%) (Fig. 2b).

\section{Thermal properties of XylE and its mutants}

As shown in Fig. 2c, the optimal temperature of XylE and its hybrid enzymes was measured over a temperature range of $60-90{ }^{\circ} \mathrm{C}$ at the respective optimal $\mathrm{pH}$. Among them, the mutants, XylE-M3, XylE-M6, XylE-M3/M6, and XylE-M3/M6/M9, had similar optimal temperature of $75{ }^{\circ} \mathrm{C}$, which was $5{ }^{\circ} \mathrm{C}$ higher than that of XylE and other mutants $\left(70{ }^{\circ} \mathrm{C}\right)$. Figure $2 \mathrm{~d}$ indicates that the $T_{50}$ value of XylE was determined as $66.5^{\circ} \mathrm{C}$, while the hybrids, XylE-M3, XylE-M3/M6, XylE-M3/M9, and XylEM3/M6/M9, had increased $T_{50}$ values of 71.2, 70.4, 70, and $69.5^{\circ} \mathrm{C}$, respectively.

To investigate the thermostability of all the enzymes, their thermodynamic stabilities $\left(T_{\mathrm{m}}\right)$ and half-lives $\left(t_{1 / 2}\right)$ at $65{ }^{\circ} \mathrm{C}$ were measured. The mutants, XylE-M3, XylE-M3/M6, XylE-M3/M9, and XylE-M3/M6/M9,

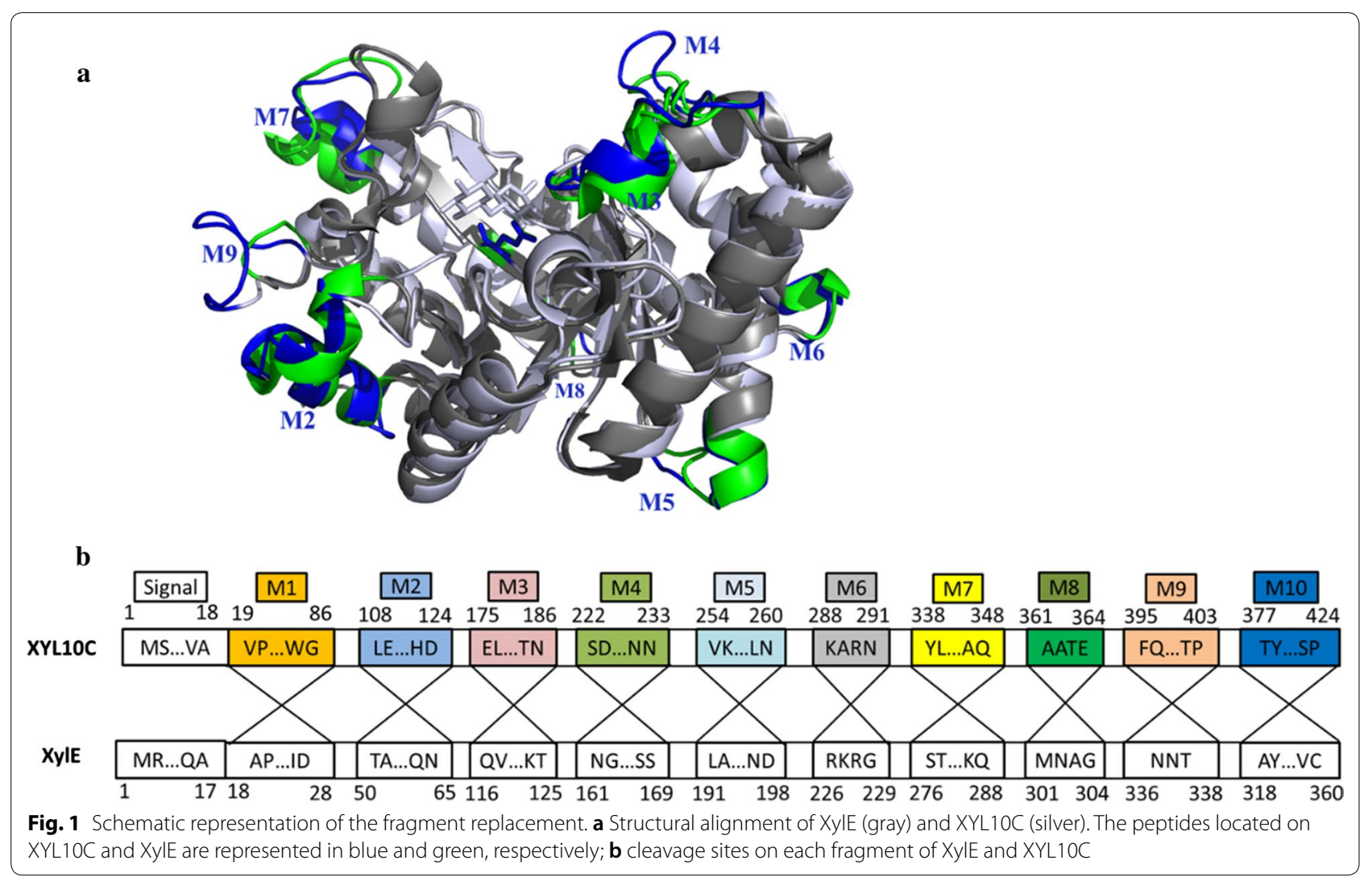



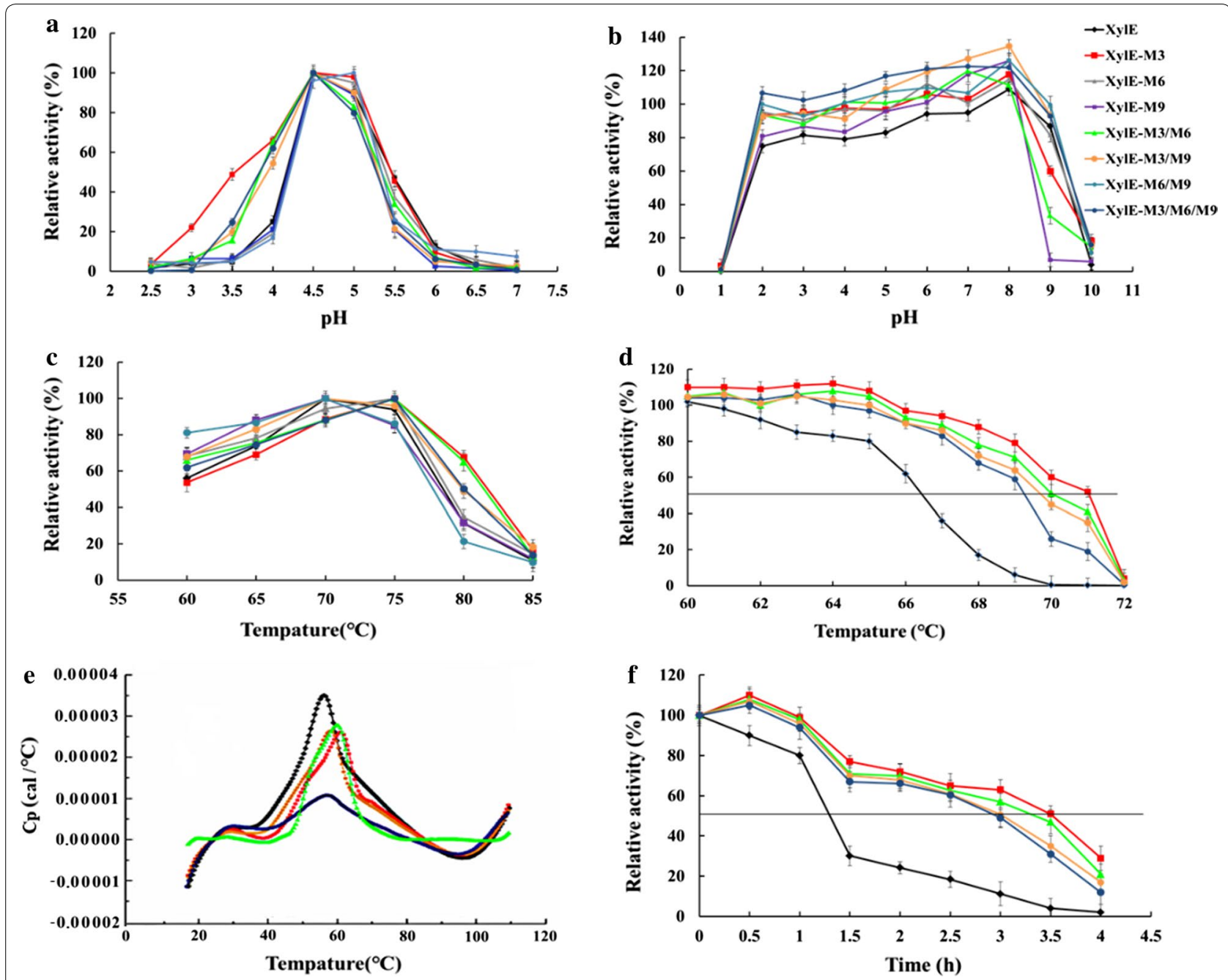

Fig. 2 Enzymatic properties of the purified recombinant wild-type XylE and its hybrid mutants. a pH activity profiles tested at the optimal temperature for each enzyme $\left(70^{\circ} \mathrm{C}\right.$ for XyIE, XyIE-M9, XylE-M3/M9, and XyIE-M6/M9; and $75^{\circ} \mathrm{C}$ for the others). $\mathbf{b} \mathrm{pH}$ stability profiles. After incubation of the enzymes at $37^{\circ} \mathrm{C}$ for $1 \mathrm{~h}$ in buffers ranging from $\mathrm{pH} 1$ to 10 , the residual activities were determined in $100 \mathrm{mM}$ Mcllvaine buffer at the optimal $\mathrm{pH}$ and optimal temperature of each enzyme. c Temperature activity profiles tested at the optimal pH of each enzyme ( $\mathrm{pH} 4.5$ for XylE and $\mathrm{pH} 5$ for the others). $\mathbf{d}$ Temperature stability profiles $\left(T_{50}\right)$. e Thermograms determined by using DSC. The calorimetric recordings for XyIE and its mutants were scanned at $1{ }^{\circ} \mathrm{C} / \mathrm{min}$ in $10 \mathrm{mM}$ phosphate-buffered saline (PBS) (pH 6.5) at $350 \mu \mathrm{g} / \mathrm{mL}$. f Half-lives of wild-type XylE and its mutants at $65^{\circ} \mathrm{C}$

had markedly improved thermostability. In comparison with the $T_{\mathrm{m}}$ and $t_{1 / 2}$ values of wild-type XylE $\left(56.1{ }^{\circ} \mathrm{C}\right.$ and $1.2 \mathrm{~h}$ ), mutants XylE-M3, XylE-M3/M6, XylE-M3/ M9, and XylE-M3/M6/M9 had increased $T_{\mathrm{m}}$ values (by approximately $4.7,3.6,2$, and $1.1^{\circ} \mathrm{C}$, respectively; Fig. 2e) and apparently longer half-lives $(1.8-2.3 \mathrm{~h}$; Fig. 2f). This result indicates that replacement of $\mathrm{M} 3$, alone or in combination, improves not only the $\mathrm{pH}$ properties, but also the thermal properties of XylE.

Specific activity and kinetics of XylE and its hybrid mutants The specific activity of all the enzymes was measured at a temperature of $70{ }^{\circ} \mathrm{C}$ and $\mathrm{pH} 5$. Among the seven active single-fragment-substituted mutants, XylE-M3, XylEM6, and XylE-M9 exhibited significantly higher specific activities compared with that of XylE (1130-1310 U/mg vs. $610 \mathrm{U} / \mathrm{mg}$ ). Using combined fragment replacement, the specific activity was further improved to $2010 \mathrm{U} / \mathrm{mg}$ (XylE-M3/M6). These results indicated that certain fragments might interact to improve enzyme catalysis.

The kinetic parameters of all enzymes were also measured with beechwood xylan as a substrate $(\mathrm{pH} 5$, $70{ }^{\circ} \mathrm{C}$ and $5 \mathrm{~min}$ ). All the graphs were plotted based on the Lineweaver-Burk regression and the equation that was used to calculate the $K_{\mathrm{m}}$ and $V_{\max }$ for each enzyme construct is shown in supplement material (Additional 
Table 1 Specific activity and kinetic parameters of XylE and its hybrid enzymes

\begin{tabular}{|c|c|c|c|c|c|}
\hline Enzymes & $K_{\mathrm{m}}(\mathrm{mg} / \mathrm{mL})$ & $V_{\max }(\mu \mathrm{mol} / \mathrm{min} / \mathrm{mg})$ & $k_{\text {cat }}(/ \mathrm{s})$ & $k_{\text {cat }} / K_{\mathrm{m}}(\mathrm{mL} / \mathrm{s} / \mathrm{mg})$ & $\begin{array}{l}\text { Specific } \\
\text { activity (U/ } \\
\text { mg) }\end{array}$ \\
\hline XYL10C & $0.54 \pm 0.02$ & $3600 \pm 66$ & $2400 \pm 16$ & $4400 \pm 131$ & $3200 \pm 101$ \\
\hline XylE & $0.75 \pm 0.04$ & $680 \pm 31$ & $430 \pm 12$ & $570 \pm 18$ & $610 \pm 18$ \\
\hline XylE-M3 & $0.61 \pm 0.03$ & $1390 \pm 41$ & $860 \pm 16$ & $1340 \pm 22$ & $1310 \pm 45$ \\
\hline XylE-M6 & $0.42 \pm 0.01$ & $1160 \pm 17$ & $740 \pm 20$ & $1760 \pm 28$ & $1150 \pm 55$ \\
\hline XylE-M9 & $0.46 \pm 0.02$ & $1390 \pm 38$ & $900 \pm 10$ & $1960 \pm 31$ & $1130 \pm 38$ \\
\hline XylE-M3/M6 & $0.62 \pm 0.02$ & $2200 \pm 28$ & $1410 \pm 22$ & $2280 \pm 29$ & $2010 \pm 51$ \\
\hline XylE-M3/M9 & $0.42 \pm 0.02$ & $880 \pm 21$ & $570 \pm 16$ & $1370 \pm 22$ & $730 \pm 21$ \\
\hline XylE-M6/M9 & $0.57 \pm 0.01$ & $1280 \pm 34$ & $820 \pm 19$ & $1440 \pm 18$ & $1270 \pm 37$ \\
\hline XylE-M3/M6/M9 & $0.67 \pm 0.03$ & $1760 \pm 47$ & $1170 \pm 26$ & $1750 \pm 19$ & $1500 \pm 46$ \\
\hline XYL10C-M & ND & ND & ND & ND & ND \\
\hline$X Y L 10 C-M_{6}$ & $2.02 \pm 0.04$ & $2530 \pm 77$ & $1890 \pm 56$ & $940 \pm 23$ & $1830 \pm 66$ \\
\hline XYL10C-M & ND & ND & ND & ND & ND \\
\hline
\end{tabular}

The specific activities mentioned were calculated after conducting assays with the enzymes at $70^{\circ} \mathrm{C}$ using beechwood xylan as the substrate $N D$ no activity detected

file 1: Figure S3). Table 1 shows that the $K_{\mathrm{m}}$ values of all the mutants were lower than that of the wild type (56-94\%), indicating their increased substrate affinity. Moreover, the mutants, XylE-M3, XylE-M6, XylE-M9, XylE-M3/M6, XylE-M3/M9, XylE-M6/M9, and XylEM3/M6/M9, showed increased catalytic efficiencies $\left(k_{\text {cat }} / K_{\mathrm{m}}\right)$, ranging from 2.4 - to fourfold, compared with those of XylE. When these three peptide segments were reversibly replaced in XYL10C, no activity was detected for the mutants XYL10C-M $M_{3}$ and XYL10C-M $M_{9}$. The catalytic efficiency and specific activity of the mutant XYL10C-M $\mathrm{M}_{6}$ were only $19 \%$ and $57 \%$ of the wild type, respectively (Table 1 ). The results indicated that these three peptide segments (M3, M6, and M9) have significant influence on the catalytic efficiency of the GH10 xylanases and can increase the catalytic efficiency and specific activity of the enzyme. In addition, combination of multiple fragments did not show a simple additive effect.

\section{Analysis of the hydrolyzed product}

High-performance liquid chromatography (HPLC) analysis indicated that XylE and its mutants, XylE-M3, XylE-M6, and XylE-M9, hydrolyzed the $\beta$-1,4-glycosidic bonds of beechwood xylan into mainly xylose and xylobiose with trace amounts of xylotriose and xylohexaose by endo-mode of action (Additional file 1: Figure S4). The order of hydrolytic activity was XylE-M3 $>$ XylEM6 > XylE-M9 > XylE. These results corresponded to their order of catalytic efficiency, and this indicated that the replacement of the peptide segments had no effect on the catalytic mechanism of XylE and its mutants.

\section{Circular dichroism (CD) measurements}

$\mathrm{CD}$ analysis was done to check whether the enhanced catalytic efficiency of the mutant was a result of variations in the secondary structure of the protein. As depicted in Fig. 3, the far-UV CD spectrum of XylE showed a distinctive minimum and maximum at 208 and $196 \mathrm{~nm}$, and these are characteristics of $\alpha$-helix and $\beta$-sheet structures in aqueous solution, respectively [24]. Fragment substitution showed that the unchanged secondary structure of XylE-M9 with a far-UV CD spectrum was similar to XylE. Contrary to the other mutants, which only had one minimum peak near $198 \mathrm{~nm}$, this type of structure is considered irregular [25]. These results might explain the importance of fragment substitution for improving the catalytic efficiency and thermal property of the enzymes.

\section{Molecular dynamic (MD) simulation}

To further explore the substrate binding in the catalytic sites of the enzyme, the enzyme-substrate complex was

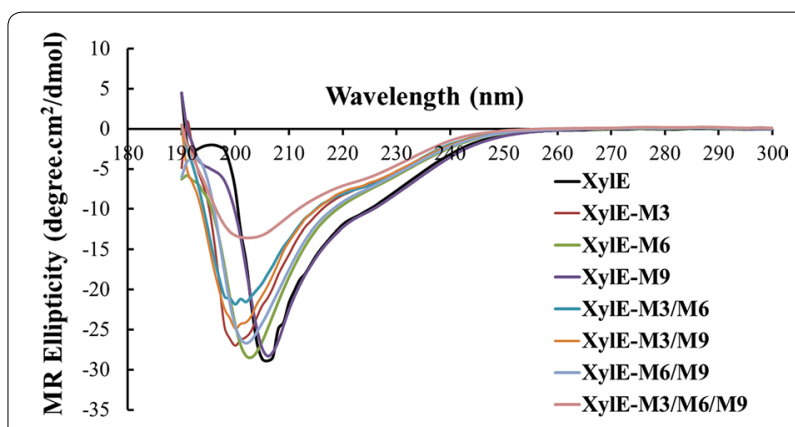

Fig. 3 Far-UV CD spectra and RMSD values of the wild-type XylE and its hybrid mutants 


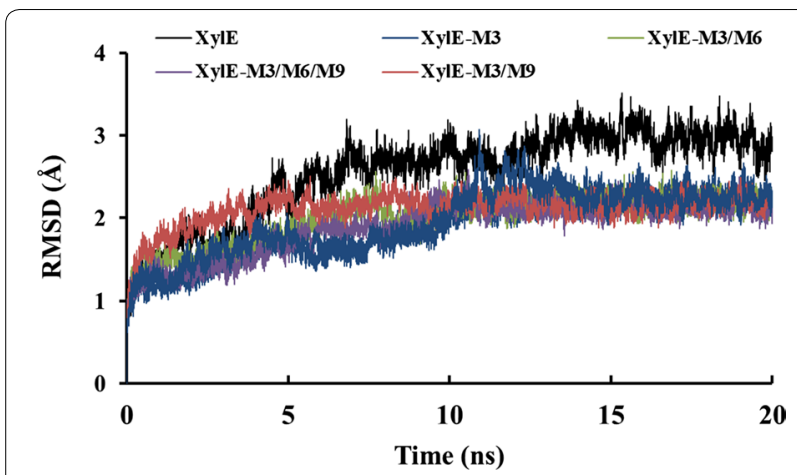

Fig. 4 RMSD values of the wild-type XylE and its hybrid mutants. The RMSD values are shown for the XyIE-M3, XylE-M3/M6, XylE-M3/ M9, and XyIE-M3/M6/M9 mutants calculated from the molecular dynamics (MD) simulation

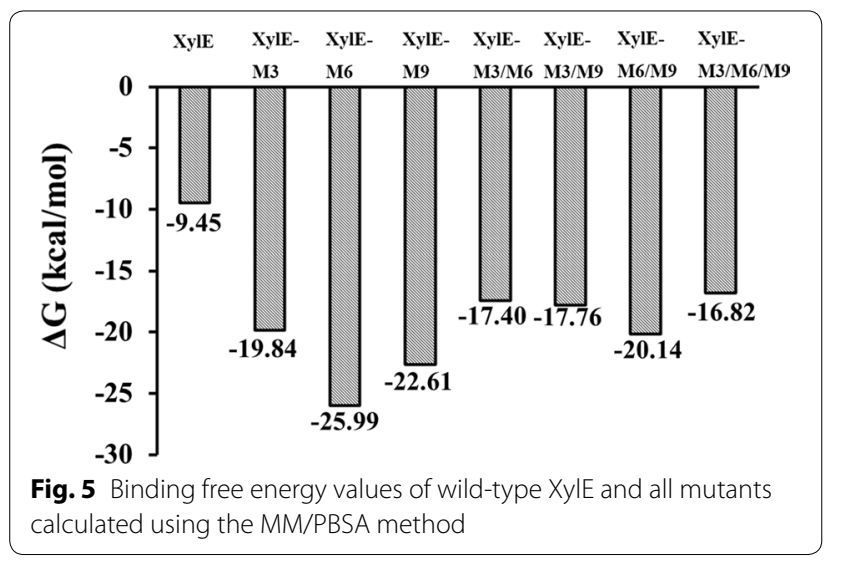

analyzed using the Discovery Studio 2017 Client. MD simulations of the wild-type XylE and hybrid mutants with the substrate molecule were used to analyze the effect of the peptide segment on ligand binding and plasticity of the protein. During the course of these MD simulations, the root mean square deviation (RMSD) of the $\alpha$-carbon atoms of all the complex systems (XylE and its seven hybrid mutants with the substrate) showed stable plateaus after 15 ns (Fig. 4 and Additional file 1: Figure S5). Compared with wild-type XylE, the mutants, XylEM3, XylE-M3/M6, XylE-M3/M9, and XylE-M3/M6/M9, showed decreased conformational flexibility, with lower RMSD values at $298 \mathrm{~K}$ (Fig. 4). These results are consistent with the experimental results, which showed that the thermostability of the four hybrids was markedly improved.

Binding free energy can be used to assess the sum of interactions between a protein and its ligand [26]. Based on the molecular mechanics Poisson-Boltzmann surface area (MM/PBSA) analysis, the binding free energies for the protein-ligand interactions of the mutants were much lower than that of XylE $(-16.82$ to $-25.99 \mathrm{kcal} /$ mol vs. $-9.45 \mathrm{kcal} / \mathrm{mol}$; Fig. 5). The result corresponded to their $K_{\mathrm{m}}$ values. This indicated higher interactions between these hybrid enzymes and their substrates, thereby enhancing their affinity to the substrate. Further hydrogen interaction analysis indicated that XylE-M3 and XylE-M9 formed nine and eight hydrogen bonds at M3 and $M 9$, respectively, whereas the wild-type XylE formed four and two hydrogen bonds at the corresponding fragments (Table 2). Moreover, XylE-M3 and XylE-M9 had seven and two hydrogen bonds, respectively, with higher occupancy rates than those of the wild type. Although XylE-M6 and XylE formed four hydrogen bonds at M6, three of the hydrogen bonds of XylE-M6 had higher occupancy rates than those of the XylE (Table 2). These results indicated that single substitution of M3, M6, and M9 increased local hydrogen-bonding interactions and contributed to the rigidity of the whole protein, resulting in enhanced substrate affinity.

\section{Hydrolysis of mulberry bark by cellulase and xylanase}

Xylanase and cellulase were added simultaneously or individually to mulberry bark to assess the generation of reducing sugars. In this study, wild type (XylE) and two mutants (XylE-M3/M6 and XylE-M3/M6/M9) were selected to study the synergistic degradation of mulberry bark by xylanase and cellulase. As shown in Fig. 6a, the production of reducing sugar increased over time in every group as expected. The amount of reducing sugars was higher when both xylanase and cellulase were added compared with the addition of cellulase only. After 15-h incubation with both xylanase and cellulase, the reducing sugar content in the groups treated with mutants, XylE, XylE-M3/M6, and XylE-M3/M6/M9, with cellulase reached 3.6, 3.9, and $4.2 \mu \mathrm{mol} / \mathrm{mL}$, respectively. Similarly, the amount of reducing sugars reached $3.2 \mu \mathrm{mol} /$ $\mathrm{mL}$ in the groups where cellulase was added individually. The above results indicated that xylanase mutants promoted cellulase-catalyzed hydrolysis of mulberry bark, and the order of strength was XylE-M3/M6 > XylE-M3/ M6/M9 > XylE. We also tested the reducing sugars that were formed as a result of catalysis by xylanase only. The amount of reducing sugars in mulberry bark catalyzed by XylE, XylE-M3/M6, and XylE-M3/M6/M9 was 0.28, 0.45, and $0.36 \mu \mathrm{mol} / \mathrm{mL}$, respectively, after $15 \mathrm{~h}$. This result indicated that ability of xylanase to hydrolyze mulberry bark was weak, and the mutant XylE-M3/M6 degraded the mulberry bark at the fastest rate, followed by mutant $\mathrm{XylE}-\mathrm{M} 3 / \mathrm{M} 6 / \mathrm{M} 9$, which is consistent with the previously determined catalytic activity. Based on the above results, it is apparent that treatment with a combination of xylanase and cellulase increases the hydrolysis of mulberry bark more efficiently than treatment with the individual 
Table 2 Comparison of the hydrogen bond quantity and occupancy rates of XylE and its hybrid mutants

\begin{tabular}{|c|c|c|c|c|c|c|c|}
\hline Fragments & Residue $^{a}$ & Acceptor & Donor & $\begin{array}{l}\text { Occupancy rate } \\
\text { (\%) }\end{array}$ & Acceptor & Donor & $\begin{array}{l}\text { Occupancy } \\
\text { rate (\%) }\end{array}$ \\
\hline \multirow[t]{8}{*}{ M3 } & 116 & S_115@OG & E_116@H & 11 & - & - & - \\
\hline & 118 & P_118@O & V_121@N & 47 & F_77@0 & S_118@HG & 45 \\
\hline & 119 & F_119@O & N_123@N & 48 & D_119@OD2 & S_123@OG & 57 \\
\hline & 120 & E_81@O & W_130@HE1 & 65 & - & - & - \\
\hline & 121 & P_118@O & F_122@H & 13 & S_118@0 & F_122@N & 28 \\
\hline & 122 & F_119@O & N_123@H & 48 & - & - & - \\
\hline & 123 & P_124@O & F_126@H & 15 & E_130@OE2 & K_124@NZ & 11 \\
\hline & 124 & P_124@O & F_126@H & 15 & - & - & - \\
\hline \multirow[t]{4}{*}{ M6 } & 201 & V_197@O & K_201@H & 91 & A_237@0 & R_201@NH1 & 83 \\
\hline & 202 & L_200@O & A_202@H & 10 & S_198@0 & K_201@N & 42 \\
\hline & 203 & E_153@OE2 & R_203@HH12 & 88 & E_153@OE1 & R_203@HH22 & 75 \\
\hline & 204 & K_201@O & N_204@N & 31 & R_201@O & G_208@H & 26 \\
\hline \multirow[t]{8}{*}{ M9 } & 336 & E_346@O & F_336@H & 81 & E_340@O & N_336@H & 64 \\
\hline & 337 & E_337@OD1 & L_345@H & 60 & - & - & - \\
\hline & 338 & P_338@O & N_342@HD22 & 10 & N_336@OD1 & F_338@HG1 & 37 \\
\hline & 339 & D_339@OD1 & N_342@HD22 & 64 & - & - & - \\
\hline & 340 & G_340@O & F_343@H & 24 & - & - & - \\
\hline & 342 & D_339@OD1 & N_342@HD22 & 64 & - & - & - \\
\hline & 343 & G_340@O & F_343@H & 24 & - & - & - \\
\hline & 344 & P_344@0 & K_60@H21 & 18 & - & - & - \\
\hline
\end{tabular}

The number of hydrogen bonds and occupancy rates are shown for the fragments $M 3, M 6$, and M9 during the last 5 ns of the trajectories

a Residue numbering corresponds to Fig. 2, in which the Ala18 of XylE is the first residue. The residues of wild-type XylE with lower occupancy rates are indicated in italic

enzyme. These results also showed that the xylanase exhibited both additive and boosting effects on the activity of cellulase.

\section{Synergistic effect of cellulase and xylanase}

In this study, we have shown the synergistic effect rather than a simple additive effect of xylanase on cellulase. The cumulative effect of xylanase on cellulase may be determined by evaluating the amount of reducing sugars produced by xylanase and cellulase treatment. In Fig. 6a, the amount of reducing sugars produced by individual treatment with cellulase was $2.9 \mu \mathrm{mol} / \mathrm{mL}$, while those after individual treatment with the xylanases, XylE, XylE-M3/ $\mathrm{M} 6$, and XylE-M3/M6/M9, were calculated as $0.28,0.45$, and $0.36 \mu \mathrm{mol} / \mathrm{mL}$, respectively. The values were significantly lower than those treated with the enzyme mixture containing a combination of all the xylanases and cellulase, which were $3.6,3.9$, and $4.6 \mu \mathrm{mol} / \mathrm{mL}$, respectively. Degree of synergy (DS) values were used to determine the detailed synergistic impact of xylanase on cellulosemediated hydrolysis of biomass. According to the definition, the larger the DS, the more the amount of reducing sugars generated from addition of xylanase into cellulase. In Fig. 6b, the DS values of two mutants were all above one after $3 \mathrm{~h}$, and $4 \mathrm{~h}$ for wild-type XylE with the highest degree of synergy of 1.3 by XylE-M3/M6, showing the synergistic effect of xylanase with cellulase. However, differences in the extent of synergy that is exhibited by the three different xylanases may well be the cause for the difference in their catalytic activities.

Furthermore, to explore the synergistic effect of xylanase on cellulase, changes in the dry weight of the substrates were determined after treating with different enzymes (Fig. 6c). Maximum loss in dry weight was shown in the mulberry bark samples treated with both mutant XylE-M3/M6 and cellulase followed by mutant $\mathrm{XylE}-\mathrm{M} 3 / \mathrm{M} 6 / \mathrm{M} 9$ and wild-type XylE with cellulase, indicating that the addition of xylanase to cellulase can speed up the cellulose breakdown more than those treated with only cellulase or xylanase. The reduction of the substrates treated with enzyme mixtures containing XylE, XylE-M3/M6/M9, and XylE-M3/M6 in synergy with cellulase was improved by $115 \%, 140 \%$, and $185 \%$, respectively, opposed to treatment with only cellulase after $24 \mathrm{~h}$. Although the production of soluble reducing sugars was not clearly detectable in the mulberry bark samples treated with only xylanase (Fig. 6a), we could clearly detect the total reduction in the dry weight (Fig. 6c). This may be due to hydrolysis of the insoluble xylans into dissolvable xylo-oligosaccharides 

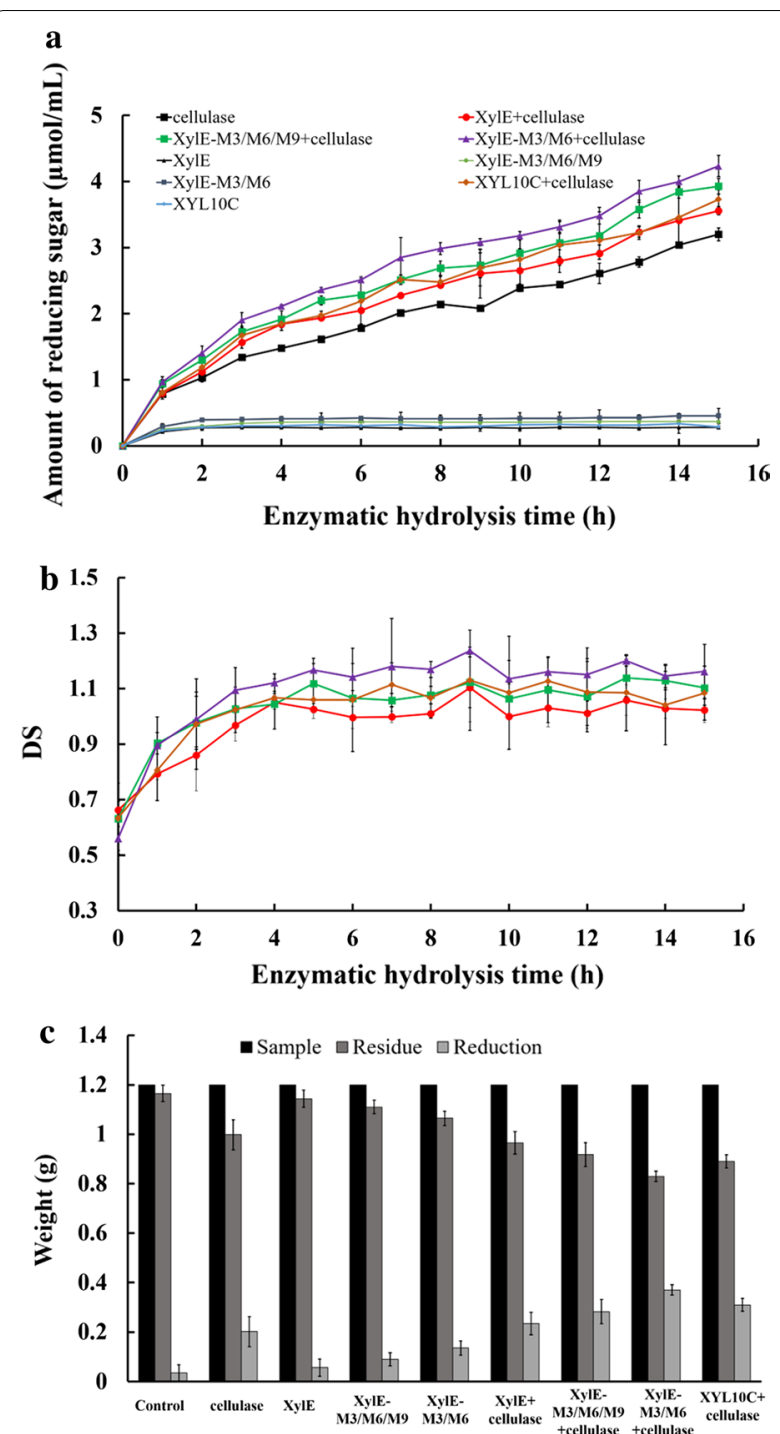

Fig. 6 Time course hydrolysis of mulberry bark. Separate hydrolysis: $5 \mathrm{U}$ each of cellulase (circle) or xylanase (triangle); simultaneous hydrolysis: $5 \mathrm{U}$ each of cellulase and xylanase (inverted triangle); control: no enzyme added (square) to substrates for $15 \mathrm{~h}$. The DS curve for the mulberry bark samples is shown. Enzyme loading: cellulase $5 \mathrm{U}$, xylanase $5 \mathrm{U}$. Control: no enzyme added. The changes in the dry weight of the mulberry bark during separate and simultaneous hydrolysis with cellulases and xylanase after $24 \mathrm{~h}$ are shown

(data not shown). We also carried out saccharification assays with XYL10C as a control as shown in Fig. 6. The experimental results showed that the synergistic effect of XYL10C with cellulase was weaker than that of XylEM3/M6 and XylE-M3/M6/M9.

\section{Scanning electron microscope (SEM) analysis}

SEM was employed to monitor the changes in the surface structure of the mulberry bark samples treated with the enzymes. From Fig. 7, it is evident that the structure of the mulberry bark treated with xylanase or cellulase changed during hydrolysis. Compared with the untreated substrate (Fig. 7a), varying degrees of damage and shedding of hemicellulose were observed on the surface of the mulberry bark treated with xylanase (Fig. 7b). After $24 \mathrm{~h}$ of treatment with cellulase alone, the microfibers in the lignocellulose separated from each other and became loose (Fig. 7c). Contrastingly, when mulberry bark was simultaneously treated with cellulase and xylanase, the microfibers were separated from the surface hemicellulose fragments and the arrangement became more chaotic, and there was more extensive disintegration of the physical structure of lignocellulose (Fig. 7d).

\section{Discussion}

Xylanases have attracted widespread attention due to their potential applications in various industries, ranging from food processing to kraft pulp bleaching [27]. The high catalytic efficiency and excellent stability of xylanases are prerequisites for their successful application in biotechnology. However, wild-type enzymes often do not have both favorable enzymatic properties and high catalytic efficiency, which limit their application in some industries [28]. In the studies attempting to improve the properties of these enzymes, most of the substitution of modules or regions were limited to the $\mathrm{N}$ terminus of the proteins $[29,30]$, and there are no reports showing the replacement of internal peptides in proteins, especially for the xylanases from the GH10 family. In addition, the catalytic efficiency of the modified xylanase produced using the site-directed mutagenesis method could not be greatly improved. Moreover, it also does not simultaneously take into account the thermostability and catalytic activity of the enzyme [31-33]. In the present study, we employed an internal peptide replacement method to construct hybrid enzymes. Compared to other enzyme engineering studies, this fragment substitution method is simple to operate, efficient for mutant construction, allows easy screening of target enzymes, and thus, represents an effective protein engineering approach.

XylE shares high amino acid sequence identity of 53\% with the highly active, acidic, and hyperthermophilic XYL10C. However, its catalytic activity is significantly lower under acidic and thermophilic conditions, which are commonly encountered in the biorefinery industry. When we replaced each fragment of XylE with its counterpart from XYL10C, seven hybrid enzymes were produced that showed xylanase activity, while three hybrids did not. This suggested that internal peptide replacement might either create novel active enzymes or produce inactive enzymes due to improper folding, even between close counterparts. Although the peptides M3, 

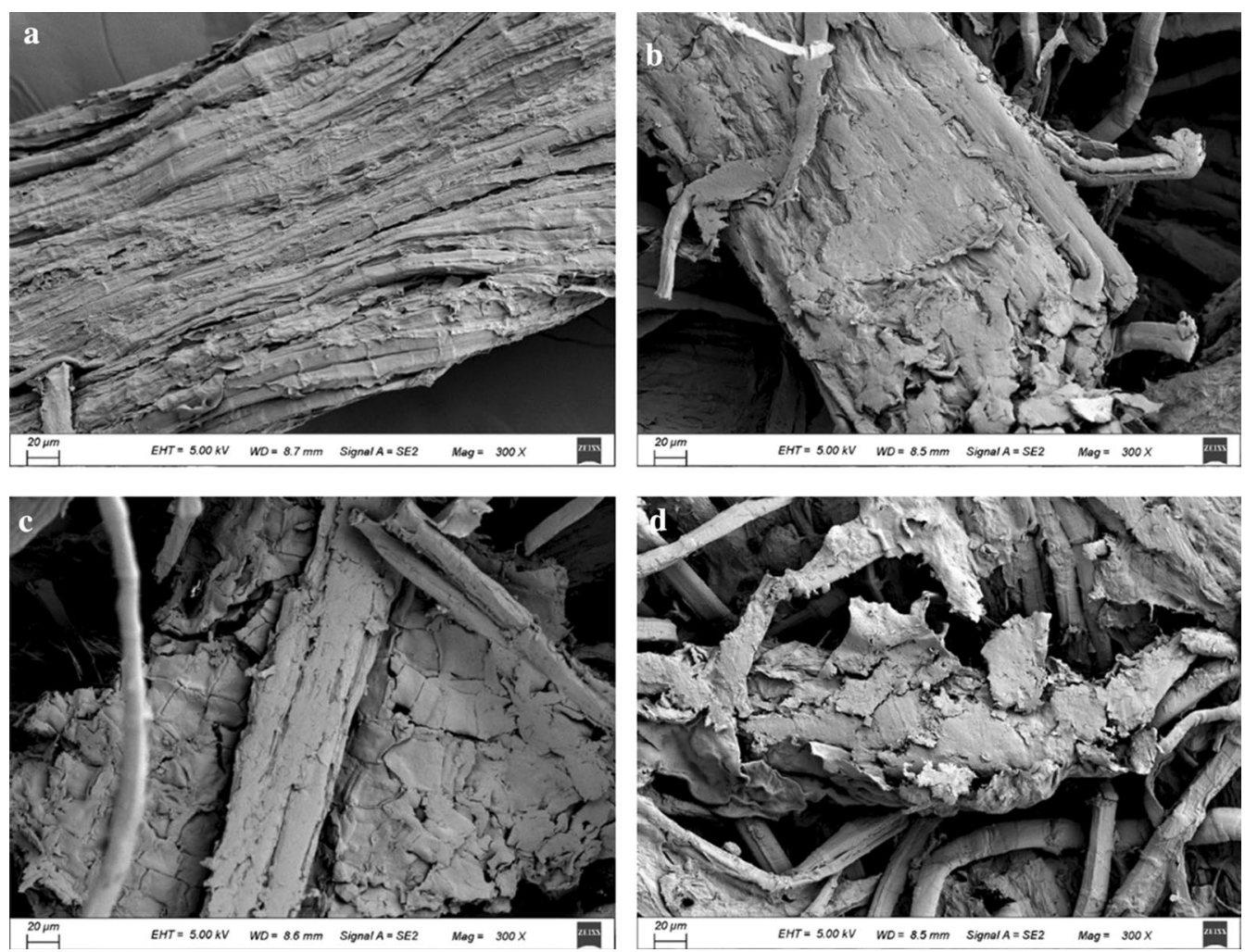

Fig. 7 Electron microscopy images of the micro structure of the mulberry bark samples treated with different enzymes. a Negative control, mulberry bark treated with buffer for $24 \mathrm{~h}$; The panels show the surface of the mulberry bark treated for $24 \mathrm{~h}$ with the following $\mathbf{b}$ XylE-M3/M6 alone; $\mathbf{c}$ cellulase alone; $\mathbf{d}$ mixture of XyIE-M3/M6 and cellulase. The magnification is 300 times larger for all images

M6, and M9 significantly contributed to the catalytic performance of XylE, substitution with their combinations had no additive effects. In general, the replacement of key peptides or residues located on the surface of a protein changes its enzymatic properties, such as the optimal conditions, catalytic efficiency, and specific activity $[14$, 34, 35]. Our structural analysis indicated that M3, M6, and M9 are all situated on the surface of XylE (Fig. 1a), and the replacement of these sequences might affect the overall structure of the protein. Hence, the improvement in the catalytic efficiency $\left(k_{\text {cat }} / K_{\mathrm{m}}\right)$ of XylE mutants can be attributed to the increase in the $V_{\max }$ and $k_{\text {cat }}$ and decrease in $K_{\mathrm{m}}$. Similar observations have been reported for proteases and phytases [36, 37].

Most enzymes show altered $\mathrm{pH}$ activity profiles at the cost of catalytic activity. For example, Yang et al. [37] constructed six mutants of a Bacillus circulans xylanase and found that three of them had a different $\mathrm{pH}$ activity range and decreased activity (up to $70 \%$ ). Given the complexity of ionic interactions, it is hard to expand the $\mathrm{pH}$ activity/adaptability profile of an enzyme through rational design [38]. Until now, most successful cases of shifting the functional $\mathrm{pH}$ range were based on random mutagenesis [39-41]. In the present study, the $\mathrm{pH}$ activity/stability profiles of XylE were improved by substitution with fragments from XYL10C. In particular, the replacement of M3 not only expanded the $\mathrm{pH}$ range in an acidic environment, but also increased the catalytic activity of the enzyme. The results indicated that the replacement of M3 might change the ionization states of the side chains of the catalytic residues [42]. Hence, our study not only improved the catalytic efficiency of XylE, but also improved its thermostability and $\mathrm{pH}$ stability. As a result, all the four hybrid enzymes, XylE-M3, XylE-M3/ M6, XylE-M3/M9 and XylE-M3/M6/M9, showed significant improvements in their specific activity, catalytic efficiency, thermostability, and/or $\mathrm{pH}$ stability.

To further investigate the effect of fragment replacement on the protein structure, MD simulation analysis of wild-type XylE and its hybrid enzymes was carried out at $298 \mathrm{~K}$ for $20 \mathrm{~ns}$. Using the RMSD value as a parameter to assess the integral structural stability of an enzyme [43], XylE, XylE-M3, XylE-M3/M6, XylE-M3/M9, and XylE-M3/M6/M9 showed marked differences compared to XylE, similar to that seen in RmXyn10A_CM, which showed enhanced thermostability and decreased RMSD 
values after deletion of the C-terminal flexible region [44]. This suggested that these four mutants probably have more rigid protein structures and this hypothesis was verified by the thermodynamic results. Theoretically, the MM/PBSA approach is a rigorous method for predicting the binding affinity of protein-substrate systems [26]. MM/PBSA analysis confirmed the impact of internal peptide substitution on the binding affinity of $\mathrm{XylE}$ and its substrates. The binding free energies of all mutants were lower than that of XylE, which indicated enhanced binding of the enzyme with the substrate favoring the catalytic process.
Molecular interactions, such as hydrogen bonds [45] and salt bridges [46], contribute to enzyme stability. MD analysis showed that more hydrogen bonds with higher occupancy rates were formed in M3, M6, and M9 of the mutants XylE-M3, XylE-M6, and XylE-M9 than in the corresponding fragment of wild-type XylE. These complex hydrogen-bonding networks might alter the overall protein structure and the interactions between the substrates and the enzymes. As shown in Fig. 8, the conformation of the substrate as well as the relative position of the catalytic channel and xylopentaose of the mutant enzymes changed markedly from that of the wild-type enzyme. In addition to playing a vital role in stabilizing

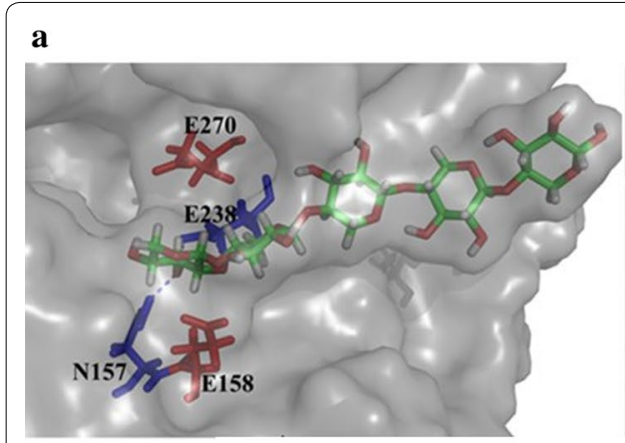

d

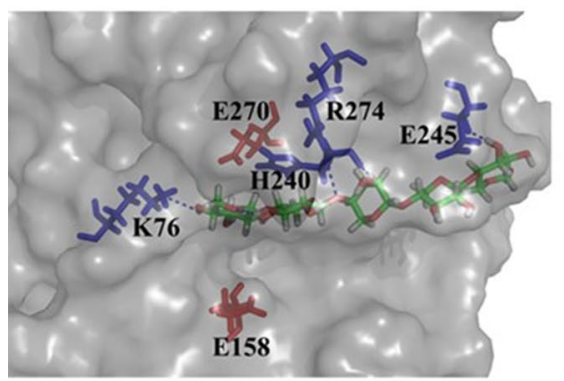

g

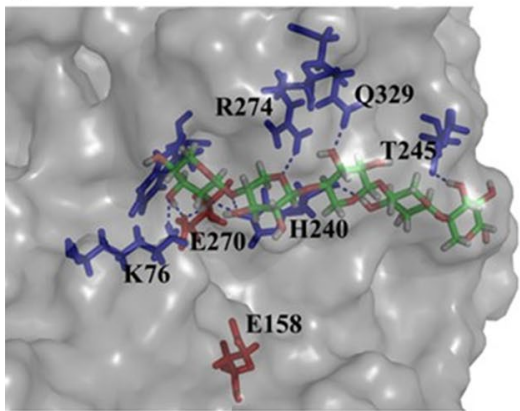

b

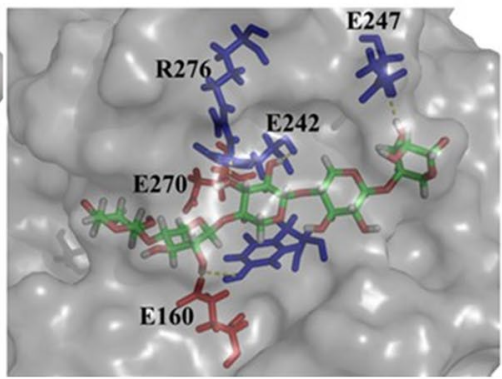

e

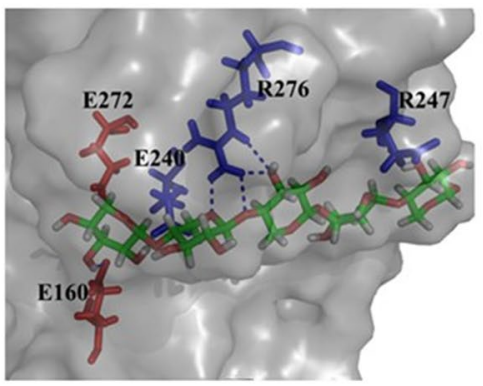

c

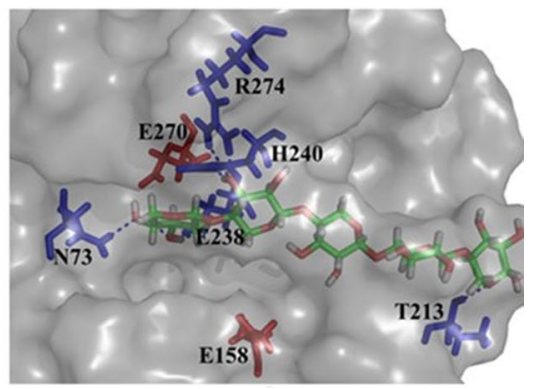

f

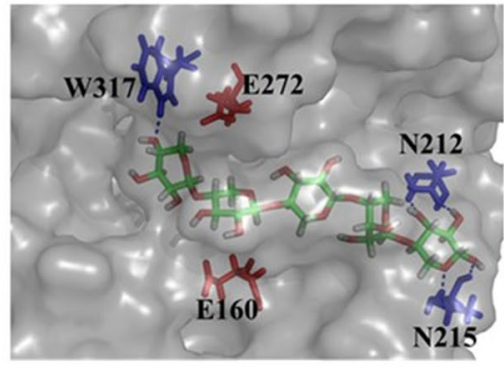

h

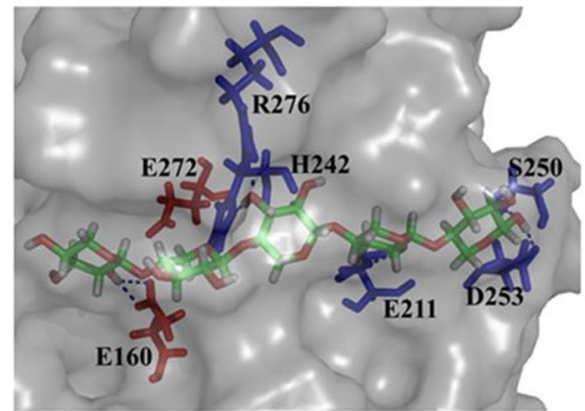

Fig. 8 Conformational changes and interactions between wild-type XylE and its hybrid mutants after binding with xylopentaose. The figure shows the confirmations and interactions between xylopentaose and the amino acid residues in the substrate binding pocket of $\mathbf{a}, \mathrm{XylE}-\mathrm{M} 3$ (b), XylE-M6 (c), XylE-M9 (d), XylE-M3/M6 (e), XyIE-M3/M9 (f), XylE-M6/M9 (g), and XylE-M3/M6/M9 (h). Catalytic residues are indicated in red; residues forming hydrogen bond interaction with the substrate are indicated in blue; xylopentaose is indicated in chromatic; and hydrogen bonds are represented by blue dashed lines 
the substrate, this type of hydrogen-bonding interaction keeps the substrate in a position that is accessible to the catalytic residues. These results further indicated that fragment replacement affects the overall structure of the enzyme and the network of the enzyme-substrate complex.

Xylanases with high catalytic activity and thermostability are required in bioenergy and biorefinery industries. Recent studies have shown that cellulases can exhibit decreased cellulose degradation rate due to adhesion to microfibrils during enzymatic hydrolysis [46]. Xylooligosaccharides induce cellulase inhibition, and the longer the xylo-oligosaccharides, the greater the inhibition [47]. Xylanase not only relieves the xylo-oligosaccharide-induced cellulase inhibition [48] but also helps in releasing the cellulases bound to the substrate [49]. Currently, most of the biorefinery processes use GH11 xylanase for industrial applications [50], and hence, the xylanase mutants obtained in this study can be potentially useful in biorefinery and bioenergy industries. Not only can these mutants synergistically degrade lignocellulosic substrates with cellulase but they can also increase the production as well as the total yield of reducing sugars. Xylanase acts as an auxiliary enzyme and destroys the physical structure of the surface of mulberry bark for easy cellulase-cellulose contact and also infiltrates the microfibril crevices of cellulose, thereby accelerating matrix breakdown. This synergistic effect of xylanase provides a feasible answer to reducing barriers in biomass degradation processes, which means that even if a minimal amount of enzymes is loaded onto the lignocellulosic substrate, higher amounts of reducing sugars can be produced. In this study, we have shown that xylanase XylE and its mutants XylE-M3/M6 and XylE-M3/M6/M9 might be able to remove xylan barriers that prevent the progressive movement of cellulases, including the cleavage of xylan into smaller chain xylo-oligosaccharides, which ultimately promotes degradation of lignocellulosic substrates [51-53]. Thus, the mutants obtained in this study could be potentially applied in biofuels, biomass degradation, and biorefineries.

\section{Conclusions}

Xylanases used in biomass degradation need to possess high catalytic efficiency and excellent thermostability. Fragment substitution method was employed to produce an immobilized xylanase with improved enzymatic properties. We observed that the three internal peptides, M3, M6, and M9, alone or in combination, notably improved the catalytic performance of XylE at higher temperatures. MD simulation and free binding energy analysis revealed that fragment replacement enhanced the local hydrogenbonding interactions and resultantly, the interaction between the substrate and the protein. We have identified the key peptides that affect the thermostability and catalytic efficiency of the GH10 xylanases. These sequences serve as useful references for studying the properties to improve the GH10 xylanase and enabling the development of an effective protein engineering strategy for potential applications in biomass degradation and bioenergy industry. The synergistic degradation experiment elucidated a possible mechanism for cellulase inhibition by xylan and xylo-oligomers, and we suggested a mechanism that explains how the addition of xylanase increases the yield of reducing sugars during the degradation of lignocellulose.

\section{Materials and methods}

The recombinant plasmids pPIC9-XYL10C and pPIC9XylE, which harbor the genes xyl10C (FJ492963) from Bispora sp. MEY-1 [13] and xylE (FJ860894) from P. canescens [22], were used in this work. Vectors PEASY-T3 and Escherichia coli Trans I-T1 were chosen for gene amplification. Pichia pastoris GS115 (Invitrogen, Carlsbad, CA) and pPIC9 (TransGen, Beijing, China) were used for protein expression. The cultivation and enzyme induction were conducted on the basis of the Pichia Expression kit instructions. The substrate beechwood xylan and standards were purchased from Sigma-Aldrich (St. Louis, MO). Restriction endonucleases, DNA polymerase, DNA purification kit, T4 DNA ligase, and endo- $\beta-N-$ acetylglucosaminidase $\mathrm{H}$ (Endo $\mathrm{H}$ ) were purchased from GenStar (Beijing, China). All other chemicals were of analytical grade.

\section{Fragment identification}

The amino acid sequences and structures of XYL10C and XylE were aligned using the ClustalW software (http:// www.clustal.org/) and rendered using the ESPript3.0 program (http://espript.ibcp.fr/ESPript/cgi-bin/ESPript.cgi) and PyMOL version 1.7.2.1 (DeLano Scientific), respectively. Two standards were used to determine the demarcation point of the fragments: (1) each peptide retains the local secondary structure and (2) except for the $\mathrm{N}$ - and $\mathrm{C}$-terminal domains, each fragment has a length of less than 20 amino acid residues.

\section{Construction of the mutants}

All the mutant genes were generated by overlap PCR using specific primers (Additional file 2: Table S1). In the first PCR step, parallel reactions were performed to amplify the target DNA fragments using the recombinant plasmids pPIC9-XYL10C and pPIC9-XylE as templates. A second PCR step was used to amplify the final 
DNA products using the products from the first PCR as templates and the primers XylE-F and XylE-R. The PCR products were digested using EcoRI and NotI, ligated into the pPIC9 expression vector, and sequenced. The combined substitution of key fragment was also conducted using specific primers as described above.

\section{Enzyme expression and purification in P. pastoris}

Wild-type XylE and all the fragment-substituted mutants were produced in P. pastoris, as described previously [13]. The induced culture was collected at $12,000 \times g$ for $15 \mathrm{~min}$ and then were concentrated using a Vivaflow 200 ultrafiltration membrane with a $10-\mathrm{kDa}$ molecular mass cutoff (Vivascience, Hannover, Germany), followed by desalting in $10 \mathrm{mM}$ Mcllvaine buffer (pH 6.5) on a fast protein liquid chromatography (FPLC) HiTrap Q Sepharose XL $5 \mathrm{~mL}$ column (GE Healthcare, Uppsala, Sweden). Proteins were eluted at $4 \mathrm{~mL} / \mathrm{min}$ flow rate with a linear gradient of $\mathrm{NaCl}(0-1 \mathrm{M})$. The fractions displaying xylanase activity were pooled and subjected to sodium dodecyl sulfate-polyacrylamide gel electrophoresis (SDS-PAGE). All the enzymes were purified to more than $95 \%$ homogeneity. Bradford assay was used to find out the protein concentration with bovine serine albumin as a standard.

\section{Biochemical characterization}

The xylanase activity was assayed by determining the release of reducing sugars from beechwood xylan using the dinitrosalicylic acid (DNS) method [54]. One unit of endo-xylanase activity $(1 \mathrm{U})$ correlates with the release of $1 \mu \mathrm{mol}$ of xylose equivalent for each minute under the valuation states of $\mathrm{pH} 4.5,70{ }^{\circ} \mathrm{C}$, and $10 \mathrm{~min}$.

The determination of the $\mathrm{pH}$ activity of the wild-type XylE along with the hybrid mutants was conducted at the optimal temperature for each enzyme for $10 \mathrm{~min}$ in $100 \mathrm{mM}$ Mcllvaine buffer ( $\mathrm{pH} 3-8)$. The $\mathrm{pH}$ stability of each enzyme was determined by measuring the residual activities under standard conditions $\left(\mathrm{pH} 5,70{ }^{\circ} \mathrm{C}\right.$, and $10 \mathrm{~min}$ ) after preincubation at $37^{\circ} \mathrm{C}$ and $\mathrm{pH} 1-10$ for $1 \mathrm{~h}$. The buffers used were $50 \mathrm{mM}$ glycine- $\mathrm{HCl}(\mathrm{pH} 1-2.5)$, $100 \mathrm{mM}$ Mcllvaine buffer (pH 3-8.5), and $50 \mathrm{mM}$ glycine- $\mathrm{NaOH}$ (pH 9-10).

The temperature for the maximal activity of the enzyme was assessed at the optimal pH (100 mM McIlvaine buffer) and at temperatures between 60 and $90{ }^{\circ} \mathrm{C}$ for $10 \mathrm{~min}$. The determination of half-lives $\left(t_{1 / 2}\right)$ of the enzymes was quantified by the remaining activities under standard conditions after different periods of incubation at 70 and $75{ }^{\circ} \mathrm{C}$ in the absence of substrate, which was followed by assaying the enzyme activity under standard conditions. To determine the $T_{50}$ (the temperature at which $50 \%$ of the maximal activity of an enzyme is retained), the enzymes $(50 \mu \mathrm{g} / \mathrm{mL})$ were incubated inside a temperature scope of $30{ }^{\circ} \mathrm{C}$ to $80{ }^{\circ} \mathrm{C}$ for $30 \mathrm{~min}$ in the absence of the substrate. Enzymes were put on ice immediately after heating for $10 \mathrm{~min}$, and the remaining activities were then estimated under the standard conditions. All reactions were performed in triplicate.

The $K_{\mathrm{m}}, V_{\max }$, and $k_{\text {cat }}$ values of the purified wildtype XylE and the hybrid mutants were carried out under standard conditions $\left(\mathrm{pH} 4.5,70{ }^{\circ} \mathrm{C}\right.$, and $\left.5 \mathrm{~min}\right)$ in $100 \mathrm{mM}$ Mcllvaine buffer containing $0.5-10 \mathrm{mg} / \mathrm{mL}$ beechwood xylan as the substrate. The records were plotted in accordance with the Lineweaver-Burk method. All reactions were conducted in triplicate.

\section{Differential scanning calorimetry (DSC) assay}

The melting temperature $\left(T_{\mathrm{m}}\right)$, which represents the thermodynamic stability of the wild-type XylE and its mutants, was determined using a MicroCal ${ }^{\mathrm{TM}}$ VP-Capillary DSC apparatus (GE Healthcare, Sweden). Each protein sample $(200 \mu \mathrm{g})$ was dissolved in $0.5 \mathrm{~mL}$ of $20-\mathrm{mM}$ McIlvaine buffer ( $\mathrm{pH}$ 6.5) and loaded onto the capillary automatically. The treatment of the degassed protein and controls was done at a heating rate of $120^{\circ} \mathrm{C} / \mathrm{h}$ over the temperature range of $25-100{ }^{\circ} \mathrm{C}$. The experiments were conducted in triplicate.

\section{$\mathrm{CD}$ analysis of the protein structure}

The spectra of proteins $(200 \mu \mathrm{g} / \mathrm{mL}$ in $10 \mathrm{mM}$ sodium acetate, $\mathrm{pH}$ 6.5) were recorded on a Chirascan CD spectrometer (Applied Photophysics, Surrey, UK) from 190 to $250 \mathrm{~nm}$, using a 1-mm cell and a bandwidth of $1 \mathrm{~nm}$ over three scans, at a scan rate of $120 \mathrm{~nm} / \mathrm{min}$. The results of circular dichroism analysis were analyzed using the convolutional neural network mastery (CDNN) processing software.

\section{Analysis of hydrolyzed product}

HPLC was used to assess the capacities of wild-type XylE and its mutants to hydrolyze xylan, as described previously [55]. Purified recombinant enzymes $(0.5 \mathrm{U})$ were incubated with $0.5 \mathrm{mg}$ of beechwood xylan in Mcllvaine buffer $(\mathrm{pH}$ $4.5)$ at $60^{\circ} \mathrm{C}$ for $30 \mathrm{~min}$. Samples $(400 \mu \mathrm{L})$ were heated in a boiling water bath for $10 \mathrm{~min}$ to terminate the reaction and then centrifuged at $12,000 \times g$ at $4{ }^{\circ} \mathrm{C}$ for $10 \mathrm{~min}$. Each sample $(30 \mu \mathrm{L})$ was injected into an HPLC instrument equipped with a Carbo PacPA100 guard column $(4 \times 50 \mathrm{~mm})$ and an analytical column $(4 \times 250 \mathrm{~mm})$. The oligosaccharides were eluted using a mobile phase of $\mathrm{NaOH}(100 \mathrm{mM})$ at a flow rate of $1 \mathrm{~mL} / \mathrm{min}$. Xylose and xylo-oligosaccharides (X2 to $\mathrm{X6)}$ were utilized as standards.

\section{Homology modeling, molecular docking, and MD simulation}

The homology modeling and structure optimization for energy minimization of all mutants were completed 
based on the X-ray structure of XylE (PDB: 4F8X) from P. canescens, performed using Discovery Studio 2017 (Accelrys Software). To analyze the enzyme and the substrate interactions, XylE and its mutants were each docked with xylopentaose conceptually using Discovery Studio 2017. The docked enzyme-substrate complexes were screened for the most reliable binding conformation, as described previously [56, 57]. The MD simulations were done utilizing the Amber 14 package at a temperature of $298 \mathrm{~K}$ for $20 \mathrm{~ns}$. Force fields ff99SB with the TIP3P water model were used to describe the systems $[58,59]$. The RMSD was determined for the protein backbone molecules utilizing minimum squares fitting. The initial 15-ns simulation was treated as the equilibration time frame, and the information of the last 5 ns was utilized for data analysis. Three-dimensional molecular visualization and figure preparation were performed using PyMOL version 1.7.2.1 and Discovery Studio 2017 Client, respectively. MM/PBSA technique was utilized to ascertain the binding free energies of the complexes of XylE or each mutant and the ligand using MD simulations [60].

\section{Enzymatic hydrolysis of mulberry bark}

Mulberry bark was pretreated with 15\% (w/w) ammonia solution at $60^{\circ} \mathrm{C}$ for $24 \mathrm{~h}$ as reported by Li et al. [61]. The pretreated mulberry bark was dissolved in $100 \mathrm{mM} \mathrm{Mcll-}$ vaine buffer $(\mathrm{pH} 5)$ at the concentration of $4 \%(\mathrm{w} / \mathrm{v})$, followed by the addition of the xylanases, XylE, XylE-M3/ M6, and XylE-M3/M6/M9, and cellulase from Aspergillus niger [62, 63]. Then, $30 \mathrm{~mL}$ of the reactants was shaken at $220 \mathrm{rpm}$ in a $50-\mathrm{mL}$ conical flask at $50{ }^{\circ} \mathrm{C}$ for $24 \mathrm{~h}$. Triplicate experiments were performed in parallel in along with a control group and the experimental groups were xylanase $(5 \mathrm{U})$-added group, cellulase $(5 \mathrm{U})$-added group, and both xylanase (5 U)-and-cellulase (5 U)-added groups. Then, $1 \mathrm{~mL}$ of hydrolyzed sample was collected at 1 -h time intervals and was used to determine the amount of reducing sugars using DNS (3,5-dinitrosalicylic acid) method.

The analysis of the DS is determined according to the formula reported originally [64], $\mathrm{DS}=Y_{1+2} /\left(Y_{1}+Y_{2}\right)$. $Y_{1+2}$ is the total amount of reducing sugar liberated during hydrolysis when adding xylanase and cellulase simultaneously. $Y_{1}$ and $Y_{2}$ represent the amount of reducing sugar liberated during hydrolysis when cellulase and xylanase are added, respectively.

\section{SEM analysis}

After reaction completion, insoluble precipitates were collected, washed with $\mathrm{ddH}_{2} \mathrm{O}$ to make sure all the soluble components are removed, and then dried at $60{ }^{\circ} \mathrm{C}$. The dried samples were sputter-coated with gold, and the surface structure was observed by a scanning electron microscope with $300 \times$ magnification.

\section{Supplementary information}

Supplementary information accompanies this paper at https://doi. org/10.1186/s13068-019-1620-7.

Additional file 1: Figure S1. Sequence alignment of XylE and XYL10C. The fragments of M1 to M10 in XYL10C are marked in different colors. Figure S2. Sodium dodecyl sulfate-polyacrylamide gel electrophoresis (SDS-PAGE) analysis of the purified recombinant wild-type XylE and its mutants. Lanes: $M$, the standard protein molecular weight markers; $A$, C, E, G, I, K, N, and P: XylE, XylE-M3, XylE-M6, XylE-M9, XylE-M3/M6, XylEM3/M9, XylE-M6/M9, and XylE-M3/M6/M9; B, D, F, H, J, L, O, and Q: the deglycosylated enzymes. Figure $\mathbf{S 3}$. Graph showing the Lineweaver and Burk regression and the equation that was used to calculate $K_{m}$ and $V_{\max }$ for each enzymatic construction. A to J represent XylE, XylE-M3, XylE-M6, XylE-M9, XylE-M3/M6, XylE-M3/M9, XylE-M6/M9, and XylE-M3/M6/M9, respectively. Figure $\mathbf{S} 4$. High-performance liquid chromatography (HPLC) analysis of the hydrolysis products of beechwood $x y l a n$ produced by the XylE (A) and its mutants. X1, xylose; X2, xylobiose; X3, xylotriose; X4, xylotetraose; X5, xylopentaose; and X6, xylohexaose. Figure S5. Root mean square deviation (RMSD) values of the wild-type XylE and its hybrid mutants XylE-M6, XylE-M9, and XylE-M6/M9 during the molecular dynamiCs (MD) simulation.

Additional file 2: Table S1. Primers used in this study.

\section{Abbreviations}

BMGY: buffered glycerol complex medium; BMMY: buffered methanol complex medium; CD: circular dichroism; DNS: 3,5-Dinitrosalicylic acid; DS: degree of synergy; DSC: differential scanning calorimetry; Endo $\mathrm{H}$ : endo- $\beta-N$ acetylglucosaminidase $\mathrm{H}$; HPLC: high-performance liquid chromatography; MD: molecular dynamic; MM/PBSA: molecular mechanics/Poisson-Boltzmann surface area; RMSD: root mean square deviation; SDS-PAGE: sodium dodecyl sulfate-polyacrylamide gel electrophoresis; SEM: scanning electron microscope.

\section{Acknowledgements}

Not applicable.

\section{Authors' contributions}

SY performed the major experiments including the construction of mutants and enzyme characterization and drafted the manuscript; $C X$ and $Y G$ participated in enzyme activity assay; $\mathrm{RM}, \mathrm{HH}, \mathrm{AR}$, and XS participated in the discussion and revised the manuscript; $H C$ and $Y B$ performed data analysis and guided the experiment. JW and HL were the corresponding authors; they designed the study, supervised the work, and analyzed the experimental data. All authors reviewed the results. All authors read and approved the final manuscript.

\section{Funding}

This research was supported by the Natural Science Foundation of Jiangsu Province (BK20190957), the National Natural Science Foundation of China (Grant Number 21978121), the National Natural Science Foundation of China (Grant Number 31472127), the Collaborative Innovation Project of Chinese Academy of Agricultural Sciences (Grant Number CAAS-XTCX2016011-04-5), and the National Chicken Industry Technology System of China (Grant Number CARS-41). Thanks to Editage for helping us to polish the language of the article.

\section{Availability of data and materials}

The dataset supporting the conclusions of this article is included within the article (and its Additional files 1 and 2).

Ethics approval and consent to participate

Not applicable. 


\section{Consent for publication}

All authors consent the manuscript for publication in Biotechnology for Biofuels.

\section{Competing interests}

The authors declare that they have no competing interests.

\section{Author details}

${ }^{1}$ Key Laboratory for Feed Biotechnology of the Ministry of Agriculture, Feed Research Institute, Chinese Academy of Agricultural Sciences, Beijing 100081 China. ${ }^{2}$ School of Biotechnology, Jiangsu University of Science and Technology, Zhenjiang 212018, People's Republic of China. ${ }^{3}$ Sericultural Research Institute, Chinese Academy of Agricultural Sciences, Zhenjiang 212018, People's Republic of China.

Received: 20 August 2019 Accepted: 25 November 2019 Published online: 03 December 2019

\section{References}

1. Thanapal SS, Annamalai K, Sweeten JM, Gordillo SG. Fixed bed gasification of dairy biomass with enriched air mixture. Appl Energy. 2012:97:525-31.

2. Sornkade P, Atong D, Sricharoenchaikul V. Conversion of cassava rhizome using an in situ catalytic drop tube reactor for fuel gas generation. Renew Energy. 2015;79:38-44.

3. Pan X, Kadla JF, Ehara K, Gilkes N, Saddler JN. Organosolv ethanol lignin from hybrid poplar as a radical scavenger: relationship between lignin structure, extraction conditions, and antioxidant activity. J Agric Food Chem. 2006:54(16):5806-13.

4. Mohanty SM, Behera S, Swain MR, Ray RC. Bioethanol production from mahula (Madhuca latifolia L.) flowers by solid-state fermentation. Appl Energy. 2009;86:640.

5. Gírio FM, Fonseca C, Carvalheiro F, Duarte LC, Marques S, BogelŁukasik R. Hemicelluloses for fuel ethanol: a review. Bioresour Technol. 2010;101(13):4775-800.

6. Balat $\mathrm{M}$, Balat $\mathrm{H}$. Recent trends in global production and utilization of bio-ethanol fuel. Appl Energy. 2009;86(11):2273-82.

7. Wijma HJ, Floor RJ, Janssen DB. Structure- and sequence-analysis inspired engineering of proteins for enhanced thermostability. Curr Opin Struct Biol. 2013;23(4):588-94.

8. Lilyan C, Cross HF, She JK, Gabriel C, Martins HFP, Cameron N. Covalent attachment of proteins to solid supports and surfaces via Sortase-mediated ligation. PLoS ONE. 2007;2(11):e1164.

9. Park HJ, Park K, Yong HK, Yoo YJ. Computational approach for designing thermostable Candida antarctica lipase B by molecular dynamics simulation. J Biotechnol. 2014;192:66-70.

10. Deshmukh RA, Jagtap S, Mandal MK, Mandal SK. Purification, biochemical characterization and structural modelling of alkali-stable $\beta-1,4$-xylan xylanohydrolase from Aspergillus fumigatus R1 isolated from soil. BMC Biotechnol. 2016;16(1):11.

11. Enkhbaatar B, Lee $C$, Hong Y, Hong S. Molecular characterization of xylobiose- and xylopentaose-producing $\beta$-1,4-endoxylanase SCO5931 from Streptomyces coelicolor A3(2). Appl Biochem Biotechnol. 2016;180(2):1-12

12. Guo N, Zheng J, Tian J, Wu L, Zhou H. Characterization and constitutive

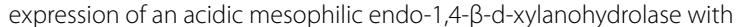
high thermotolerance and catalytic efficiency in Pichia pastoris. World J Microbiol Biotechnol. 2013;29(11):2095-103.

13. Luo HY, Li J, Yang J, Wang H, Yang YH, Huang HQ, Shi PJ, Yuan TZ, Fan YL, Yao B. A thermophilic and acid stable family-10 xylanase from the acidophilic fungus Bispora sp. MEY-1. Extremophiles. 2009;13(5):849-57.

14. Wang Y, Feng SY, Zhan T, Huang ZQ, Wu GJ, Liu ZD. Improving catalytic efficiency of endo- $\beta-1,4-x y l a n a s e$ from Geobacillus stearothermophilus by directed evolution and $\mathrm{H} 179$ saturation mutagenesis. J Biotechnol. 2013;168(4):341-7.

15. Wang X, Ge H, Zhang D, Wu S, Zhang G. Oligomerization triggered by foldon: a simple method to enhance the catalytic efficiency of lichenase and xylanase. BMC Biotechnol. 2017;17(1):57.

16. Hoseki J, Okamoto A, Takada N, Suenaga A, Futatsugi N, Konagaya A, et al. Increased rigidity of domain structures enhances the stability of a mutant enzyme created by directed evolution. Biochemistry. 2003;42(49):14469-75.

17. Lee SG, Hong SP, Song JJ, Kim SJ, Sung MH. Functional and structural characterization of thermostable D-amino acid aminotransferases from Geobacillus spp. Appl Environ Microbiol. 2006;72(2):1588-94.

18. Christopher LP, Kumar H, Zambare VP. Enzymatic biodiesel: challenges and opportunities. Appl Energy. 2014;119(12):497-520.

19. Mehta SC, Rice K, Palzkill T. Natural variants of the KPC-2 carbapenemase have evolved increased catalytic efficiency for ceftazidime hydrolysis at the cost of enzyme stability. PLoS Pathog. 2015;11(6):e1004949.

20. Zheng F, Huang H, Wang X, Tu T, Liu Q, Meng K, et al. Improvement of the catalytic performance of a Bispora antennata cellulase by replacing the $\mathrm{N}$-terminal semi-barrel structure. Bioresour Technol. 2016;218:279-85.

21. Song L, Dumon C, Siguier B, André I, Eneyskaya E, Kulminskaya A, et al. Impact of an N-terminal extension on the stability and activity of the GH11 xylanase from Thermobacillus xylanilyticus. J Biotechnol. 2014;174:64-72.

22. Fedorova TV, Chulkin AM, Vavilova EA, Maisuradze IG, Trofimov AA, Zorov IN, et al. Purification, biochemical characterization, and structure of recombinant endo-1,4- $\beta$-xylanase XyIE. Biochemistry. 2012;77(10):1190-8.

23. You $S$, Chen $C$, Tu T, Wang X, Ma R, Cai H, et al. Insight into the functional roles of Glu175 in the hyperthermostable xylanase XYL10C- $\triangle N$ through structural analysis and site-saturation mutagenesis. Biotechnol Biofuels. 2018;11(1):159.

24. Kelly SM, Jess TJ, Price NC. How to study proteins by circular dichroism. Biochim Biophys Acta. 2005;1751(2):119-39.

25. Provencher SW, Gl Ckner J. Estimation of globular protein secondary structure from circular dichroism. Biochemistry. 1981;20(1):33-7.

26. Tingjun $H$, Junmei $W$, Youyong $L$, Wei W. Assessing the performance of the MM/PBSA and MM/GBSA methods. 1. The accuracy of binding free energy calculations based on molecular dynamics simulations. J Chem Inf Model. 2011;51(1):69-82.

27. Polizeli MLTM, Rizzatti ACS, Monti R, Terenzi HF, Jorge JA, Amorim DS. Xylanases from fungi: properties and industrial applications. Appl Microbiol Biotechnol. 2005;67(5):577-91.

28. Porter JL, Rusli RA, Ollis DL. Directed evolution of enzymes for industrial biocatalysis. ChemBioChem. 2016;17(3):197-203.

29. Yang TC, Legault S, Kayiranga EA, Kumaran J, Ishikawa K, Sung WL. The $\mathrm{N}$-terminal $\beta$-sheet of the hyperthermophilic endoglucanase from Pyrococcus horikoshii is critical for thermostability. Appl Environ Microbiol. 2012;78:3059-67.

30. Wang $K$, Luo $H$, Bai Y, Shi P, Huang $H$, Xue X, Yao B. A thermophilic endo-1,4- $\beta$-glucanase from Talaromyces emersonii CBS394.64 with broad substrate specificity and great application potentials. Appl Microbiol Biotechnol. 2014;98:7051-60.

31. Zhang JF, Shi H, Xu LY, Zhu XY, Li XQ. Site-directed mutagenesis of a hyperthermophilic endoglucanase Cel12B from Thermotoga maritima based on rational design. PLOS ONE. 2015;10(10):e0141937.

32. Cheng YS, Chen CC, Huang JW, Ko TP, Huang ZY, Guo RT. Improving the catalytic performance of a $\mathrm{GH} 11$ xylanase by rational protein engineering. Appl Microbiol Biotechnol. 2015:99(22):9503-10.

33. Lin L, Hu S, Yu K, Huang J, Yao S, Lei Y, et al. Enhancing the activity of glutamate decarboxylase from Lactobacillus brevis by directed evolution. Chin J Chem Eng. 2014;22(11-12):1322-7.

34. Jia H, Li Y, Liu Y, Yan Q, Yang S, Jiang Z. Engineering a thermostable $\beta-1,3-1,4-g l u c a n a s e$ from Paecilomyces thermophila to improve catalytic efficiency at acidic pH. J Biotechnol. 2012;159(1-2):50-5.

35. Trollope KM, Görgens JF, Volschenk H. Semirational directed evolution of loop regions in Aspergillus japonicus $\beta$-fructofuranosidase for improved fructooligosaccharide production. Appl Environ Microbiol. 2015;81(20):7319-29.

36. Chen W, Ye L, Guo F, Lv Y, Yu H. Enhanced activity of an alkaline phytase from Bacillus subtilis 168 in acidic and neutral environments by directed evolution. Biochem Eng J. 2015;98(15):137-43.

37. Ji HY, Park JY, Kim SH, Yoo YJ. Shifting pH optimum of Bacillus circulans xylanase based on molecular modeling. J Biotechnol. 2008;133(3):294-300

38. Shunchen $\mathrm{Q}$, Luhua $\mathrm{L}$. Tailoring the $\mathrm{pH}$ dependence of human nonpancreatic secretory phospholipase A2 by engineering surface charges. Appl Biochem Biotechnol. 2013;171(6):1454-64. 
39. Yuqi Q, Xiaomin W, Xin S, Yinbo Q. Engineering endoglucanase II from Trichoderma reesei to improve the catalytic efficiency at a higher $\mathrm{pH}$ optimum. J Biotechnol. 2008;135(2):190-5.

40. Fan C, Xu W, Zhang T, Zhou L, Jiang B, Mu W. Engineering of Alicyclobacillus hesperidum I-arabinose isomerase for improved catalytic activity and reduced $\mathrm{pH}$ optimum using random and site-directed mutagenesis. Appl Biochem Biotechnol. 2015;177(7):1480-92.

41. Ma F, Xie Y, Luo M, Wang S, Hu Y, Liu Y. Sequence homolog-based molecular engineering for shifting the enzymatic pH optimum. Synth Syst Biotechnol. 2016;1(3):195-206.

42. Cockburn DW, Clarke AJ. Modulating the $\mathrm{pH}$-activity profile of cellulase a from Cellulomonas fimi by replacement of surface residues. Protein Eng Des Sel. 2011;24(5):429-37.

43. Chen J, Yu H, Liu C, Liu J, Shen Z. Improving stability of nitrile hydratase by bridging the salt-bridges in specific thermal-sensitive regions. J Biotechnol. 2013;164(2):354-62.

44. Aronsson A, Güler F, Petoukhov MV, Crennell SJ, Svergun DI, Linares-Pastén JA, Nordberg Karlsson E. Structural insights of RmXyn10A-a prebiotic-producing GH10 xylanase with a non-conserved aglycone binding region. Biochim Biophys Acta Proteins Proteom. 2017:1866(2):292-306.

45. Vogt G, Woell S, Argos P. Protein thermal stability, hydrogen bonds, and ion pairs. J Mol Biol. 1997;269(4):631-43.

46. Haney P, Konisky J, Koretke KK, Luthey-Schulten Z, Wolynes PG. Structural basis for thermostability and identification of potential active site residues for adenylate kinases from the archaeal genus Methanococcus. Proteins. 1997;28(1):117-30.

47. Igarashi K, Uchihashi T, Koivula A, Wada M, Kimura S, Okamoto T, et al. Traffic jams reduce hydrolytic efficiency of cellulase on cellulose surface. Science. 2011;333(6047):1279-82.

48. Kumar R, Wyman CE. Effect of xylanase supplementation of cellulase on digestion of corn stover solids prepared by leading pretreatment technologies. Bioresour Technol. 2009;100(18):4203-13.

49. Qing Q, Wyman CE. Supplementation with xylanase and $\beta$-xylosidase to reduce xylo-oligomer and xylan inhibition of enzymatic hydrolysis of cellulose and pretreated corn stover. Biotechnol Biofuels. 2011:4(1):1-12.

50. Eriksson T, Börjesson J, Tjerneld F. Mechanism of surfactant effect in enzymatic hydrolysis of lignocellulose. Enzyme Microb Technol. 2002;31(3):353-64.

51. Henrissat B, Davies G. Structural and sequence-based classification of glycoside hydrolases. Curr Opin Struct Biol. 1997;7(5):637-44.

52. Zhang J, Siikaaho M, Puranen T, Ming T, Tenkanen M, Viikari L. Thermostable recombinant xylanases from Nonomuraea flexuosa and Thermoascus aurantiacus show distinct properties in the hydrolysis of xylans and pretreated wheat straw. Biotechnol Biofuels. 2011;4(1):12.

53. Kolenová K, Biely P. Mode of action of endo-beta-1,4-xylanases of families 10 and 11 on acidic xylooligosaccharides. J Biotechnol. 2006;121(3):338-45.

54. Miller GL. Use of dinitrosalicylic acid reagent for determination of reducing sugar. Anal Biochem. 1959;31(3):426-8.

55. Xue X, Wang R, Tu T, Shi P, Ma R, Luo H, et al. The N-terminal GH10 domain of a multimodular protein from Caldicellulosiruptor bescii is a versatile xylanase/ $\beta$-glucanase that can degrade crystalline cellulose. Appl Environ Microbiol. 2015;81(11):3823.

56. Sandgren M, Berglund GI, Shaw A, Ståhlberg J, Kenne L, Desmet T. Crystal complex structures reveal how substrate is bound in the -4 to the +2 binding sites of Humicola grisea Cel12A. J Mol Biol. 2004;342(5):1505-17.

57. Takuya Y, Toshiyuki S, Hisashi H, Mamoru S, Hiroshi H. Structural basis for inhibition of xyloglucan-specific endo- $\beta$-1,4-glucanase (XEG) by XEGprotein inhibitor. J Biol Chem. 2012;287(22):18710-6.

58. Jorgensen WL, Chandrasekhar J, Madura JD, Impey RW, Klein ML. Comparison of simple potential functions for simulating liquid water. J Chem Phys. 1983;79(2):926-35.

59. Lindorff LK, Piana SK, Maragakis P, Klepeis JL, Dror RO, Shaw DE. Improved side-chain torsion potentials for the Amber ff99SB protein force field. Proteins. 2010;78(8):1950-8.

60. Genheden S, Ryde U. The MM/PBSA and MM/GBSA methods to estimate ligand-binding affinities. Expert Opin Drug Discov. 2015;10(5):449-61.

61. Li X, Kim T, Nghiem N. Bioethanol production from corn stover using aqueous ammonia pretreatment and two-phase simultaneous saccharification and fermentation (TPSSF). Bioresour Technol. 2010;101(15):5910-6.

62. Song HT, Gao Y, Yang YM, Xiao WJ, Liu SH, Xia WC, et al. Synergistic effect of cellulase and xylanase during hydrolysis of natural lignocellulosic substrates. Bioresour Technol. 2016;219:710-5.

63. Moreau RA, Johnston DB, Powell MJ, Hicks KB. A comparison of commercial enzymes for the aqueous enzymatic extraction of corn oil from corn germ. J Am Oil Chem Soc. 2004;81(11):1071-5.

64. Jia L, Gonçalves GA, Takasugi Y, Mori Y, Noda S, Tanaka T, et al. Effect of pretreatment methods on the synergism of cellulase and xylanase during the hydrolysis of bagasse. Bioresour Technol. 2015;185:158-64.

\section{Publisher's Note}

Springer Nature remains neutral with regard to jurisdictional claims in published maps and institutional affiliations.
Ready to submit your research? Choose BMC and benefit from:

- fast, convenient online submission

- thorough peer review by experienced researchers in your field

- rapid publication on acceptance

- support for research data, including large and complex data types

- gold Open Access which fosters wider collaboration and increased citations

- maximum visibility for your research: over 100M website views per year

At BMC, research is always in progress.

Learn more biomedcentral.com/submissions 